Article

\title{
Spectral Shaping of a Superluminescent Diode for Terahertz Cross-Correlation Spectroscopy
}

\author{
Kai-Henning Tybussek ${ }^{1,2}$, Kevin Kolpatzeck ${ }^{2}{ }^{\oplus}$, Vladyslav Cherniak ${ }^{2}{ }^{\oplus}$, Sebastian Engelbrecht ${ }^{1}$, Bernd Fischer ${ }^{1}$ \\ and Jan C. Balzer $2, *$ (D) \\ 1 French-German Research Institute of Saint-Louis ISL, 68301 Saint-Louis, France; \\ Kai-Henning.Tybussek@uni-due.de (K.-H.T.); Sebastian.Engelbrecht@isl.eu (S.E.); Bernd.Fischer@isl.eu (B.F.) \\ 2 Chair of Communication Systems NTS, University of Duisburg-Essen, 47057 Duisburg, Germany; \\ Kevin.Kolpatzeck@uni-due.de (K.K.); Vladyslav.Cherniak@uni-due.de (V.C.) \\ * Correspondence: Jan.Balzer@uni-due.de
}

check for updates

Citation: Tybussek, K.-H.;

Kolpatzeck, K.; Cherniak, V.;

Engelbrecht, S.; Fischer, B.; Balzer, J.C. Spectral Shaping of a

Superluminescent Diode for

Terahertz Cross-Correlation

Spectroscopy. Appl. Sci. 2022, 12,

1772. https://doi.org/

10.3390/app12041772

Academic Editor: John Xiupu Zhang

Received: 7 December 2021

Accepted: 5 February 2022

Published: 9 February 2022

Publisher's Note: MDPI stays neutral with regard to jurisdictional claims in published maps and institutional affiliations.

Copyright: (c) 2022 by the authors. Licensee MDPI, Basel, Switzerland. This article is an open access article distributed under the terms and conditions of the Creative Commons Attribution (CC BY) license (https:// creativecommons.org/licenses/by/ $4.0 /)$.

\section{Featured Application: Terahertz Cross-Correlation Spectroscopy.}

\begin{abstract}
After 20 years of terahertz cross-correlation spectroscopy (THz-CCS), the performance of the systems has been improved to practical relevance by reaching a bandwidth of $\sim 2 \mathrm{THz}$. For the development of high-performance THz-CCS systems, it is necessary to get a deeper knowledge of the signal generation from incoherent light sources. In this work, the bandwidth, dynamic range, and peak-to-peak amplitude of a THz-CCS systems using a superluminescent diode as light source and a programmable optical filter for spectral shaping was investigated to obtain a better understanding of the relationship between the optical spectrum and the generated terahertz spectrum. By a periodic structuring of the continuous optical spectrum, an enhancement of the peak dynamic range of more than $10 \mathrm{~dB}$ was achieved with a bandwidth of $1.6 \mathrm{THz}$. The experimental results are confirmed by numerical simulations.
\end{abstract}

Keywords: terahertz cross-correlation spectroscopy; pulse shaping; terahertz systems

\section{Introduction}

Since terahertz cross-correlation spectroscopy (THz-CCS) was introduced in 2000, the researchers dedicated to this research field have made significant progress towards reasonable performance improvements for practical applications. The first step towards compact THz-CCS systems was presented by Morikawa et al. (2000), using a commercially available multimode laser diode (MMLD) and photoconductive antennas (PCAs) [1]. This was the first time that a MMLD was used for broadband terahertz generation instead of an ultrafast mode-locked laser to create terahertz radiation. However, the free-space transmission in the optical path makes this system not suitable for mobile applications. Inserting single-mode fiber and a fiber-coupled 50:50 beam splitter into the optical path instead of a free space 50:50 beam splitter improved the signal-to-noise ratio (SNR) and the compactness for THz-CCS systems [2,3]. This technique for terahertz generation was later extended and renamed to terahertz quasi time-domain spectroscopy (THz-QTDS) [4]. THz-QTDS has the disadvantage of discrete frequency components, much lower bandwidth, and lower dynamic range compared to state-of-the-art terahertz time-domain spectroscopy (THz-TDS) systems. Within the last ten years researchers tried to overcome these disadvantages with different methods.

One of these methods was the implementation of a spatial light filter in the optical path to increase the continuous signal components of the terahertz spectrum by additional mode filtering. This further enhanced the SNR towards practical relevance [5]. Another solution to get a continuous terahertz spectrum using two spectrally overlapping semiconductor laser sources was presented by Molter et al. (2011). The group used two modulation techniques 
in combination, which resulted in a continuous terahertz spectrum [6]. Another issue that led to advances in THz-QTDS systems was the use of $1550 \mathrm{~nm}$ communications technology components. Fiber-coupled components from optical communications engineering can be easily implemented in THz-QTDS systems with a center wavelength of $1550 \mathrm{~nm}$. A fully fiber-coupled THz-QTDS system has been demonstrated by Kohlhaas et al. (2017) with a bandwidth of $1.8 \mathrm{THz}$ and a single shot peak dynamic range of $60 \mathrm{~dB}$. This was a huge step towards compact and mobile terahertz systems and had a bandwidth improvement of $50 \%$ [7]. All these systems used a MMLD as proposed by Morikawa et al. in 2000. The first use of monolithic-mode-locked laser diodes (MLLD) for THz-TDS as a light source was presented by Merghem et al. in 2017 [8]. This light source creates periodic pulse trains in the time-domain and discrete components in the frequency-domain. The terahertz frequency components have the same free spectral range as the laser modes. This system achieved a bandwidth of $0.6 \mathrm{THz}$ with a peak dynamic range of $45 \mathrm{~dB}$.

A method for further enhancing the performance of THz-QTDS systems regarding the bandwidth is the realization of a low duty cycle of the injection current of the MMLD. Pulsing the injection current showed an increase of the bandwidth [9]. The possibility of optical feedback of the laser diode was presented one year later by Rehn et al. (2019). The group could raise the bandwidth of the system from $1.8 \mathrm{THz}$ to $2.5 \mathrm{THz}$ with optical feedback [10]. These improvements bring the performance level of modern THz-QTDS systems to practical relevance and therefore the research towards more compact systems matured as well. Tonder et al. (2019) presented a compact and inexpensive THz-QTDS system for mobile scenarios with a bandwidth of $\sim 0.8 \mathrm{THz}$ [11].

Until recently, it was always a laser source that illuminates the PCAs or the photomixers of a terahertz system. In 2019, Molter et al. showed that THz-QTDS systems can also be driven by an incoherent light source. The used light source was a superluminescent diode (SLD), which is normally used in optical coherence tomography because of its broad spectrum and short coherence length. In THz-QTDS systems, this light source creates a continuous spectrum in the terahertz frequency-domain, while the rest of the setup is comparable to a conventional THz-TDS system. It was demonstrated that the bandwidth of such a THz-QTDS system scales linearly with the optical bandwidth of the light source until it saturates around $1.8 \mathrm{THz}$. This was demonstrated by using different optical bandpass filters and analyzing the resulting terahertz spectrum [12]. However, none of these techniques can overcome all the disadvantages of semiconductor-based light sources-driven terahertz systems. Although theories exist for THz-CCS with an incoherent light source, they are not complete. For MMLDs, the phase relation and linewidth of the modes are neglected [4]. This leads to improper simulation results. As an example, the envelope of quasi pulse train is not reproduced, which is an effect of the finite linewidth. A better understanding of the principles is mandatory for the further development of these systems.

To get a better understanding of the behavior of terahertz generation with photomixers driven by an incoherent light source, we present in this work an experimental setup for indirect terahertz pulse shaping. This is done by manipulating the optical spectrum of a SLD with special interest in system performance improvement. Dynamic range, peak-topeak amplitude, terahertz power and bandwidth are investigated depending on the shape and optical power of the incident optical signal. We show that we can further improve these parameters with optical shaping and amplification of the optical spectrum. The shaping is realized by a programmable optical filter (POF).

In the following section, the experimental setup and the different experiments are explained in detail. We created 65 different filter shapes differing in the distance between the features and the width of the features. As a next step, the corresponding terahertz spectrum was analyzed, and important parameters were extracted and are presented in Section 3. At the end of Section 3, the results are compared to a numerical simulation. 


\section{Experimental Setup}

\subsection{THz-CCS System}

In this section, we explain the system configuration which was used for the indirect optical terahertz pulse shaping. The experimental setup is depicted in Figure 1. The system is split into four parts: light source system, terahertz system, electronic system for data acquisition, and the power supply unit (PSU). This section will focus on the light source system part, which is highlighted in red. It contains all the system components for manipulating and shaping of the terahertz spectrum. The terahertz and electronic system are like a conventional THz-CCS system. The light source is a commercially available SLD (SLD1550P-A40, Thorlabs, Maisons-Laffitte, France) which was also used by Molter et al. [12]. The injection current for the experiments was $\mathrm{I}_{\text {diode }}=600 \mathrm{~mA}$. The typical optical output power at a current of $600 \mathrm{~mA}$ is $50 \mathrm{~mW}$. For the optical shaping, a programmable optical filter (POF, Finisar Wave Shaper 1000A) and for amplification an erbium-doped fiber amplifier (EDFA, EDFA100P, Thorlabs, Maisons-Laffitte, France) were used. The POF has an operating wavelength range from $1526 \mathrm{~nm}$ to $1568.7 \mathrm{~nm}$ and it can attenuate frequency components by up to $35 \mathrm{~dB}$ with a step size of $0.01 \mathrm{~dB}$. The EDFA has a maximum output power of $23 \mathrm{dBm}$ and an operating wavelength range from $1530 \mathrm{~nm}$ to $1565 \mathrm{~nm}$. In front of the terahertz system part, the resulting optical spectrum is analyzed by splitting the optical power in $99 \%$ and $1 \%$ and sending $1 \%$ to an optical spectrum analyzer (OSA MS9740A, Anritsu, Kanagawa, Japan). After that, the signal gets split by a 50:50 beam splitter to the emitter on the one side and to the receiver on the other side. The receiver signal gets delayed in the free space part of a fiber-coupled mechanical delay line (OZ ODL-650-ML), which has a travel range of $50 \mathrm{~mm}(=330 \mathrm{ps})$, in a continuous scanning mode. The emitter is a InGaAs p-i-n photodiode, and the receiver is a photomixer for continuous wave excitation [13]. Both antennas are designed for an excitation wavelength of $1550 \mathrm{~nm}$. In the terahertz path, four TPX-lenses (TPX35, Menlo, Mountain View, CA, USA) are used to guide the terahertz radiation from the emitter to the receiver. For alignment of the path, a terahertz frequency-domain (THz-FDS) system (Terascan 1550, Toptica, Munich, Germany) was used. This enables a qualitative comparison of system performance in different operating modes. The data acquisition (DAQ) used for the experiments is based on a Zürich Instruments MLFI $5 \mathrm{MHz}$ lock-in amplifier. The integration time was set to $1 \mu \mathrm{s}$ and the bias for the emitter to $1.4 \mathrm{~V}$ peak-to-peak amplitude with $-0.2 \mathrm{~V}$ offset at a modulation frequency of $11.25 \mathrm{kHz}$ which are the recommended settings from the manufacturer.

\subsection{Experiment Setup}

For the experiment, 65 different bandpass filters and comb-like structures were created for the POF and amplified afterwards to the constant optical powers of 10, 15 and $20 \mathrm{~mW}$ at each antenna (for a detailed list of all filters, see Table 1). The power levels were chosen in a way that all filters can be amplified to the same maximum optical power for all experiments; 100 terahertz time traces were recorded and averaged in the time-domain to increase the SNR. After that, a Blackman window and a fast-Fourier transform was applied to the data, to get the frequency-domain representation. In the following we will give an overview of the treatment of our measured data. Therefore, the optical spectrum, the terahertz time-domain, and the corresponding terahertz spectrum are shown. Important parameters will be highlighted.

In Figure 2c, a continuous bandpass filter with the size $\Delta \mathrm{BP}=2 \mathrm{THz}$ was applied to the SLD. The performance for each filter was determined by measuring dynamic range (DR) and peak-to-peak amplitude $(\Delta p p)$. From this data, a value for the qualitative comparison of detected terahertz power $\left(P_{T H z}\right)$ and bandwidth (BW) was derived. In Figure $2 \mathrm{a}$, the recorded terahertz time-domain trace is presented and the parameter $\Delta \mathrm{pp}$ is indicated. The continuous spectrum of the SLD leads to a single terahertz pulse. Figure $2 \mathrm{~b}$ shows the corresponding terahertz spectrum. Indicated in the picture are the parameters for the peak DR specified at $\sim 200 \mathrm{GHz}$ and the range which was used for linearly fitting the slope 
$\mathrm{m}$ of the spectrum. To show the influence on the BW, the slope $\mathrm{m}$ was considered for the range between $0.2 \mathrm{THz}$ and $1.2 \mathrm{THz}$ as a parameter for the roll-off. This measurement shows that an increased DR increases the bandwidth of the system when the slope $m$ stays constant. In red, the linear fit for this measurement can be seen in Figure $2 \mathrm{~b}$. The BW was determined by this fit as the intersection between the noise floor and the continued linear fit. The noise floor was calculated as the arithmetic average of the frequency-domain signal for frequencies between 2.2-4.0 THz. The result of this method is an approximated bandwidth which makes the bandwidth parameter quantitatively comparable for the following experiments.

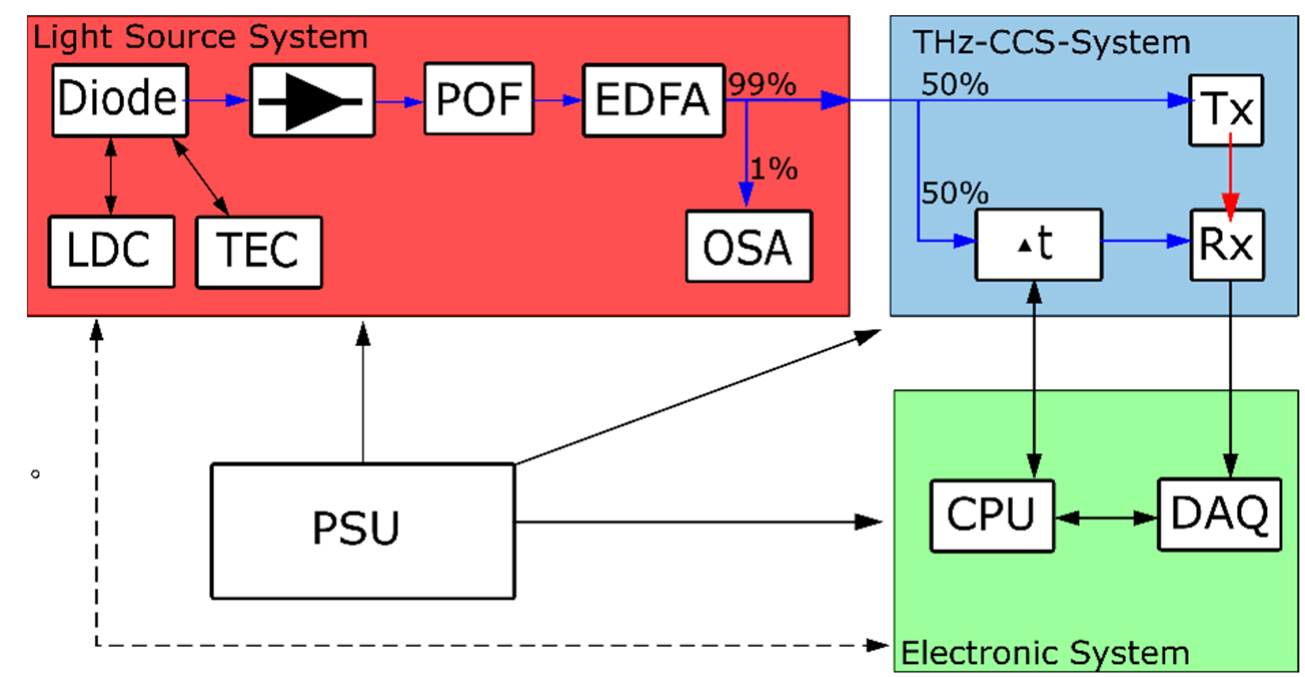

Figure 1. System overview, the light source system part in the red shaded area is the relevant part for the presented experiments. Blue arrows: optical signal; black arrows: electrical signal; red arrows: terahertz signal; dashed black arrow: control signal; diode: superluminescent diode (SLD); optical isolator; programmable optical filter (POF); erbium-doped fiber amplifier (EDFA); optical spectrum analyzer (OSA) and the light source electronics, laser diode controller (LDC) and thermal electric cooler (TEC). In blue, standard THz-TDS components are used with a photomixer as the emitter. In green: lock-in amplifier for data acquisition (DAQ).

Table 1. Overview of all created filters for the POF and the corresponding antenna power for the measurements.

\begin{tabular}{ccccc}
\hline Optical Power & Feature Width & Shapes & $\boldsymbol{\Delta f}$ & Stepsize \\
\hline $10,15,20 \mathrm{~mW}$ & $2-3 \mathrm{THz}$ & 5 & - & $200 \mathrm{GHz}$ \\
$10,15,20 \mathrm{~mW}$ & $\sim 5 \mathrm{THz}$ & 1 & - & - \\
$10,15,20 \mathrm{~mW}$ & $5 \mathrm{GHz}$ & 20 & $30-125 \mathrm{GHz}$ & $5 \mathrm{GHz}$ \\
$10,15,20 \mathrm{~mW}$ & $10 \mathrm{GHz}$ & 20 & $30-125 \mathrm{GHz}$ & $5 \mathrm{GHz}$ \\
$10,15,20 \mathrm{~mW}$ & $20 \mathrm{GHz}$ & 20 & $30-125 \mathrm{GHz}$ & $5 \mathrm{GHz}$ \\
\hline
\end{tabular}

In Figure 3c, one of the investigated optical comb structures, created with the POF, is depicted. The previously shown $2 \mathrm{THz}$ continuous bandpass filter was shaped into periodic features with a filter period $\Delta \mathrm{f}$. The filter period describes the distance between the middle of two adjacent features. For the experiment, 20 different filter periods were created with 3 different feature widths, 5,10 and $20 \mathrm{GHz}$, and $\Delta \mathrm{f}$ was varied from $30 \mathrm{GHz}$ to $125 \mathrm{GHz}$ in $5 \mathrm{GHz}$ steps (cf. Table 1). Here, the feature width describes the width of the passband of the POF which might deviate from the actual FWHM. Due to the limited resolution of our OSA, we were not able to measure the exact FWHM of the optical features. 
a)

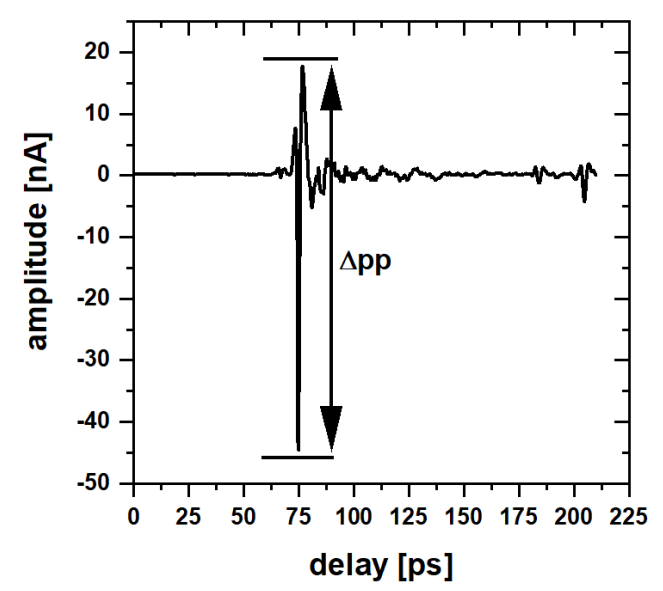

c)

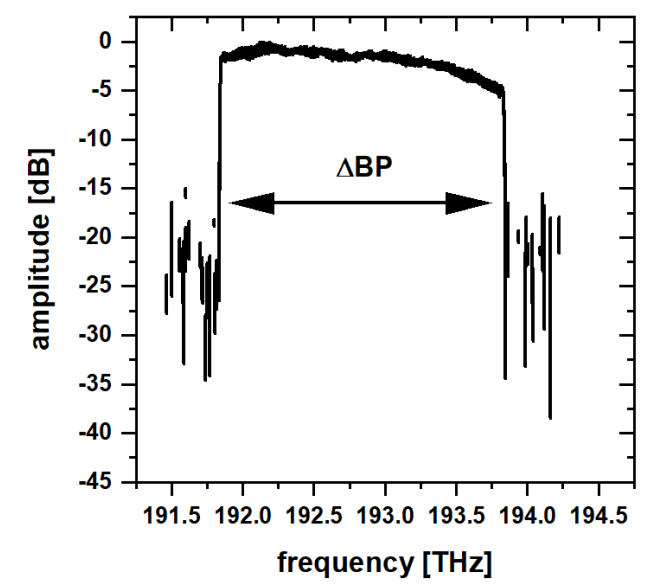

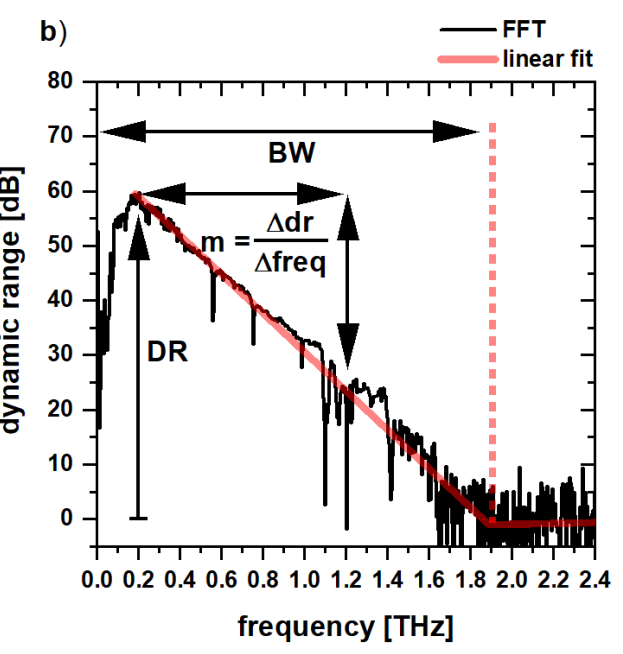

Figure 2. (a) Terahertz time-domain trace with a $\Delta p p=62.5 \mathrm{nA}$ and a FWHM $=0.9 \mathrm{ps}$. (b) Terahertz spectrum after applying a Blackman window and the FFT algorithm to the time-domain with $\mathrm{m}=-35 \mathrm{~dB} / \mathrm{THz}$ and a peak dynamic range at $200 \mathrm{GHz} \mathrm{DR}=59.5 \mathrm{~dB}$. (c) Optical spectrum from the SLD after applying a bandpass filter $\triangle \mathrm{BP}=2 \mathrm{THz}$.

A typical terahertz time-domain trace is depicted in Figure 3a. As expected for a THzQTDS trace, it consists of equally spaced pulses with a maximum peak-to-peak amplitude at the zero-delay position of the cross-correlation measurement. From here, the peak-topeak amplitude decreases due to the incoherent nature of the used light source. To quantify the amplitude variation, we calculate the envelope of the signal (red and green) and use the FWHM of the lower envelope as a parameter for the coherence. Figure $3 b$ depicts the terahertz spectrum which was retrieved by a Blackman window in the time-domain and FFT. The slope parameter was calculated from a linear fit through the detected peaks of the spectrum in the range between $0.2 \mathrm{THz}$ and $1.2 \mathrm{THz}$. 

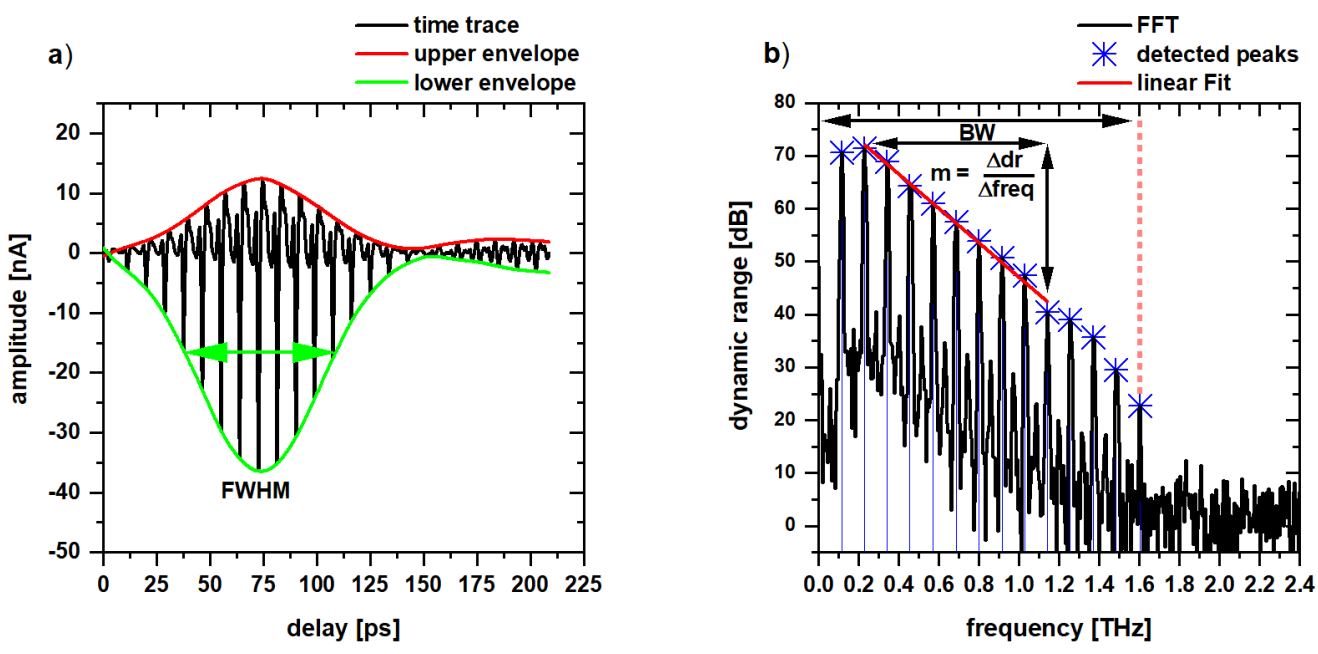

c)

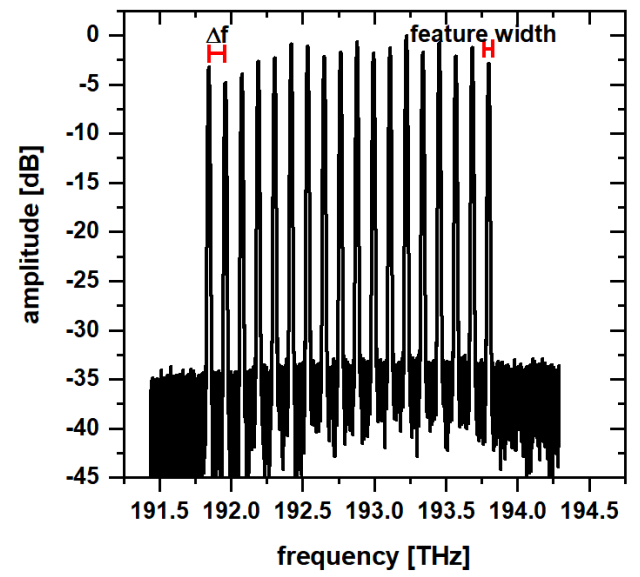

Figure 3. (a) Terahertz time-domain trace of a spectrally structured SLD. The envelope (green) has a FWHM $=66.45$ ps. (b) Frequency-domain representation of the trace with a linear fit (red) through the detected peaks (blue). The roll-off is $\mathrm{m}=-32.5 \mathrm{~dB} / \mathrm{THz}$ with a peak DR of $72 \mathrm{~dB}$ and a bandwidth of $1.6 \mathrm{THz}$. (c) Optical spectrum of the SLD after a structured $2 \mathrm{THz}$ bandpass filter with a feature spacing $\Delta \mathrm{f}=115 \mathrm{GHz}$ and a feature width of $10 \mathrm{GHz}$.

\section{Results and Discussion}

In this section, the previously defined performance parameters BW, DR, and $\mathrm{m}$ will be used to characterize the influence of the bandwidth of a continuous optical spectrum and the optical power on the terahertz spectrum. In a next step, we will use the previously defined filters (cf. Table 1) to investigate the influence of structured optical spectra on the terahertz spectrum. In the last part of this section, a simple model is presented which reproduces the measurement results.

\subsection{Analysis and Comparison of Continuous Spectra}

First, measurements with a continuous bandpass filter were done to find out the optimal size $\triangle \mathrm{BP}$ and position as a reference for the comb structured filters. There is a slight positive trend for longer excitation wavelength visible (cf. Figure A1), which can be explained by a better conversion efficiency of the low energy photons [14]. However, since the SLD has the maximum in amplified spontaneous emission around $193 \mathrm{THz}$, the region around $193 \mathrm{THz}$ was chosen for further experiments. Measurements with different continuous bandpass sizes from $0.2 \mathrm{THz}$ up to $5 \mathrm{THz}$ showed a saturation effect of the generated terahertz bandwidth around $2 \mathrm{THz}$ for a bandpass size larger or equal to $2 \mathrm{THz}$ (cf. Figure A2). This was also observed by Molter et al. [12]. To investigate whether an 
optical bandwidth above $2 \mathrm{THz}$ provides benefits away from the terahertz bandwidth, we consider terahertz power in addition to the previously mentioned indicators.

The current detected in the receiver module is the cross-correlation of the incident electromagnetic field and the optical signal at the receiver after a delay $\tau[4,15]$. Because of this correlation, the average terahertz power is proportional to the power of the squared photocurrent in the detector and can be used as a qualitative comparison factor derived from the discrete time signal [16]:

$$
P_{T H z} \propto \frac{1}{N T} \sum_{n=0}^{N} I_{R x}(n T)^{2}
$$

where $P_{T H z}$ is the calculated detected terahertz power at the receiver $(R x), T$ is the time between each data point, $N$ is the number of acquired data points, and $I_{R x}$ is the current at $R x$. It should be noted here that the calculated power refers to the detected signal and is not an approximation for the actual radiated power. Nevertheless, it allows a qualitative comparison between different operating points and correlates with the actual power [16].

Figure 4 shows the parameters DR, $\triangle \mathrm{pp}, \mathrm{BW}$ and $P_{\mathrm{THz}}$ for continuous bandpass filters from $2 \mathrm{THz}$ to $3 \mathrm{THz}$ in $0.2 \mathrm{THz}$ steps. It can be seen that $\Delta \mathrm{pp}$ drops by roughly $20 \%$, the calculated power by $50 \%$, and the DR by $3 \mathrm{~dB}(\sim 50 \%)$, while the bandwidth increases just by $5 \%$ This small gain in bandwidth does not justify a broader bandpass width. For a bandpass filter width of $2 \mathrm{THz}$, nearly the entire bandwidth is converted into the terahertz signal. For this reason, a bandpass filter width of $2 \mathrm{THz}$ was chosen for the further experiments. This saturation effect can be explained using a simple picture of photomixing. At a bandwidth of $2 \mathrm{THz}$, there is more than one possible mixing product for difference frequencies below $1 \mathrm{THz}$. Above $1 \mathrm{THz}$, there is only one mixing product. So, if the optical spectrum is increased to $3 \mathrm{THz}$, there is still exactly 1 mixing product for $2 \mathrm{THz}$, while otherwise mainly mixing products with low difference frequencies are added. Combined with the frequency characteristics of the antennas used, mixed products larger than $2 \mathrm{THz}$ are lost in the noise.

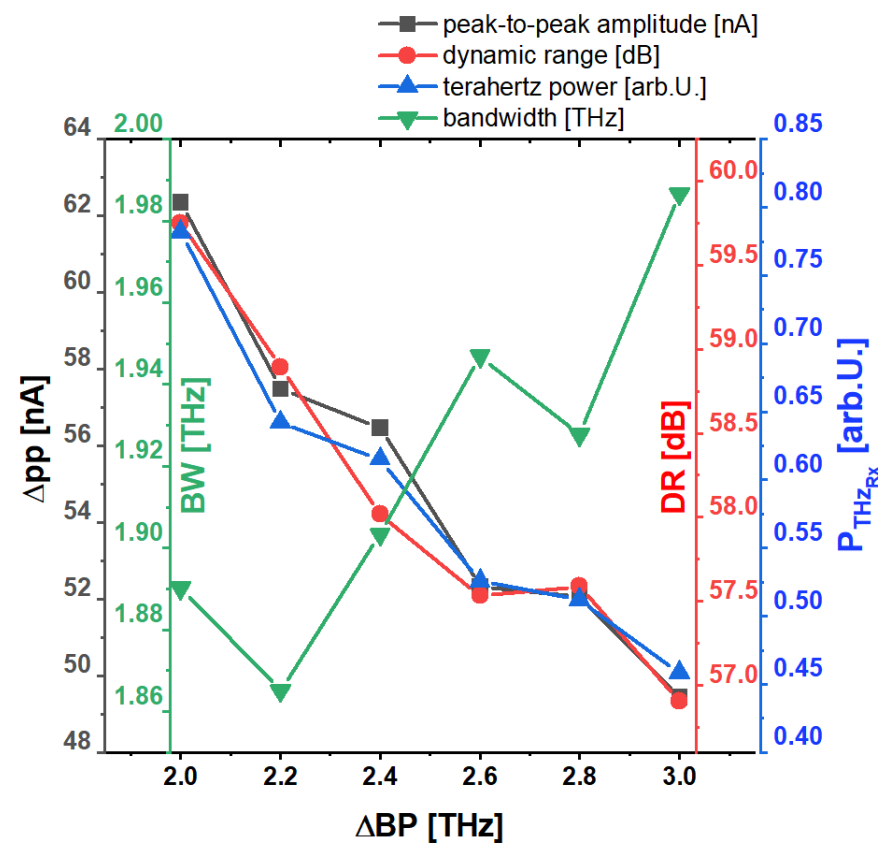

Figure 4. Different continuous bandpass widths from 2.0 to $3.0 \mathrm{THz}$ in $0.2 \mathrm{THz}$ steps and $P_{R x}=20 \mathrm{~mW}$ optical power. The three parameters DR, $\Delta \mathrm{pp}$ and $P_{T H z}$ are linearly decreasing with increasing filter width. The bandwidth parameter BW is marginally increasing for larger bandpass sizes.

As a next step, we analyzed the behavior of a $2 \mathrm{THz}$ bandpass filter for varying optical power at the antennas. The behavior between dynamic range, slope $\mathrm{m}$ and bandwidth 
for different optical powers is shown in Figure 5. In Figure 5a, the dynamic range over the incident optical power is depicted. The DR slowly saturates for increasing optical power. This can be attributed to a saturation of free carriers inside the active material of the antennas.
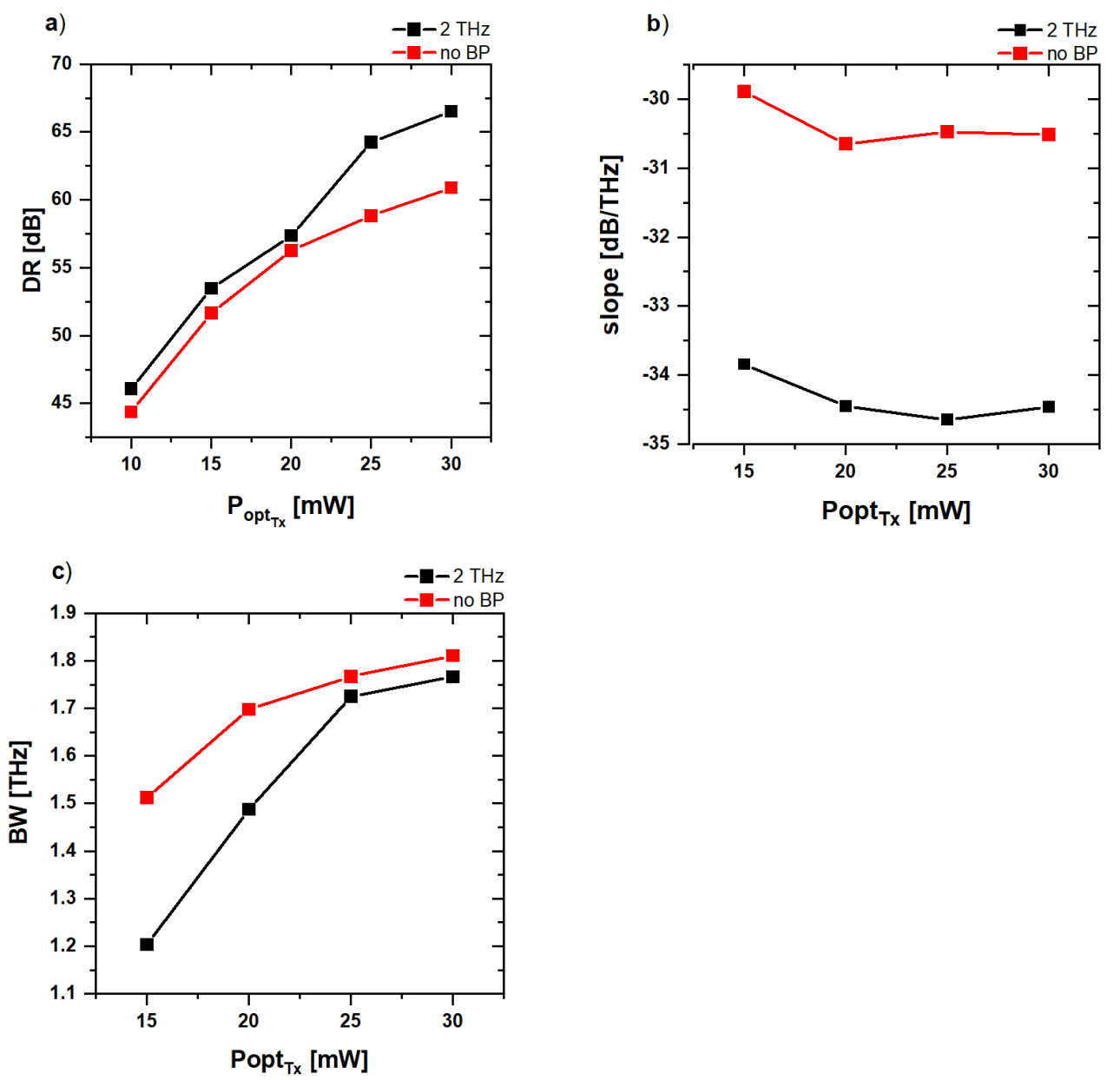

Figure 5. (a) The peak DR is slowly saturating for increased optical power. (b) The slope $m$ is slightly reduced for powers larger than $15 \mathrm{~mW}$. Powers equal to $15 \mathrm{~mW}$ or lower lead to a smaller bandwidth and a higher slope. (c) The fitted BW parameter is saturating with increased optical power at the antennas. The improvement in BW is decreased for optical powers $>25 \mathrm{~mW}$.

The impact of the optical power on the slope $m$ is depicted in Figure $5 b$. The slope $m$ shows a $0.5 \mathrm{~dB} / \mathrm{THz}$ jump from $15 \mathrm{~mW}$ towards $20 \mathrm{~mW}$ optical power. For higher optical powers, no notable changes for $\mathrm{m}$ are observable. This indicates that the gain in bandwidth for higher optical power is only due to the increased DR. A look at the bandwidth as a function of optical power (Figure 5c) shows a notable improvement up to $25 \mathrm{~mW}$. However, since the gain of the EDFA is insufficient to amplify all spectra to $25 \mathrm{~mW}$ or $30 \mathrm{~mW}$, we decided to make the following measurements only up to $20 \mathrm{~mW}$ optical power per antenna with an optical bandwidth of $2 \mathrm{THz}$. The saturation can be explained as follows. High optical power leads to a roll-over behavior of the terahertz output power, which can be explained by a screening effect. The intense optical power generates a high density of positive and negative charge carriers, which then attenuate the external electric field [17]. This leads to a saturation of the dynamic range and the bandwidth.

\subsection{Analysis and Comparison of Structured Spectra to Continouus Spectra}

In this section, the results from the comb-like shapes as shown in Figure 3 are presented and analyzed. The shapes were created out of the $2 \mathrm{THz}$ continuous bandpass 
[(cf. Figure 2c)] by periodically shaping $5 \mathrm{GHz}, 10 \mathrm{GHz}$, and $20 \mathrm{GHz}$ broad features in the continuous bandpass. This was done by blocking the output for the considered frequency span of $30 \mathrm{GHz}$ to $125 \mathrm{GHz}$ between the features, producing a periodic comb-like spectrum [(cf. Figure 3c)]. Figure 6a-c depicts the results for $P_{T H z}, \Delta \mathrm{pp}$ and DR, respectively, for different filter periods $\Delta \mathrm{f}$ for $10 \mathrm{GHz}$ and $20 \mathrm{GHz}$ broad features at $20 \mathrm{~mW}$ incident optical power per antenna. For comparison with the continuous spectrum, the respective result for the $2 \mathrm{THz}$ bandpass is indicated as a black line. The best value for each feature width and filter period is indicated by an arrow and the value is written in the figure.
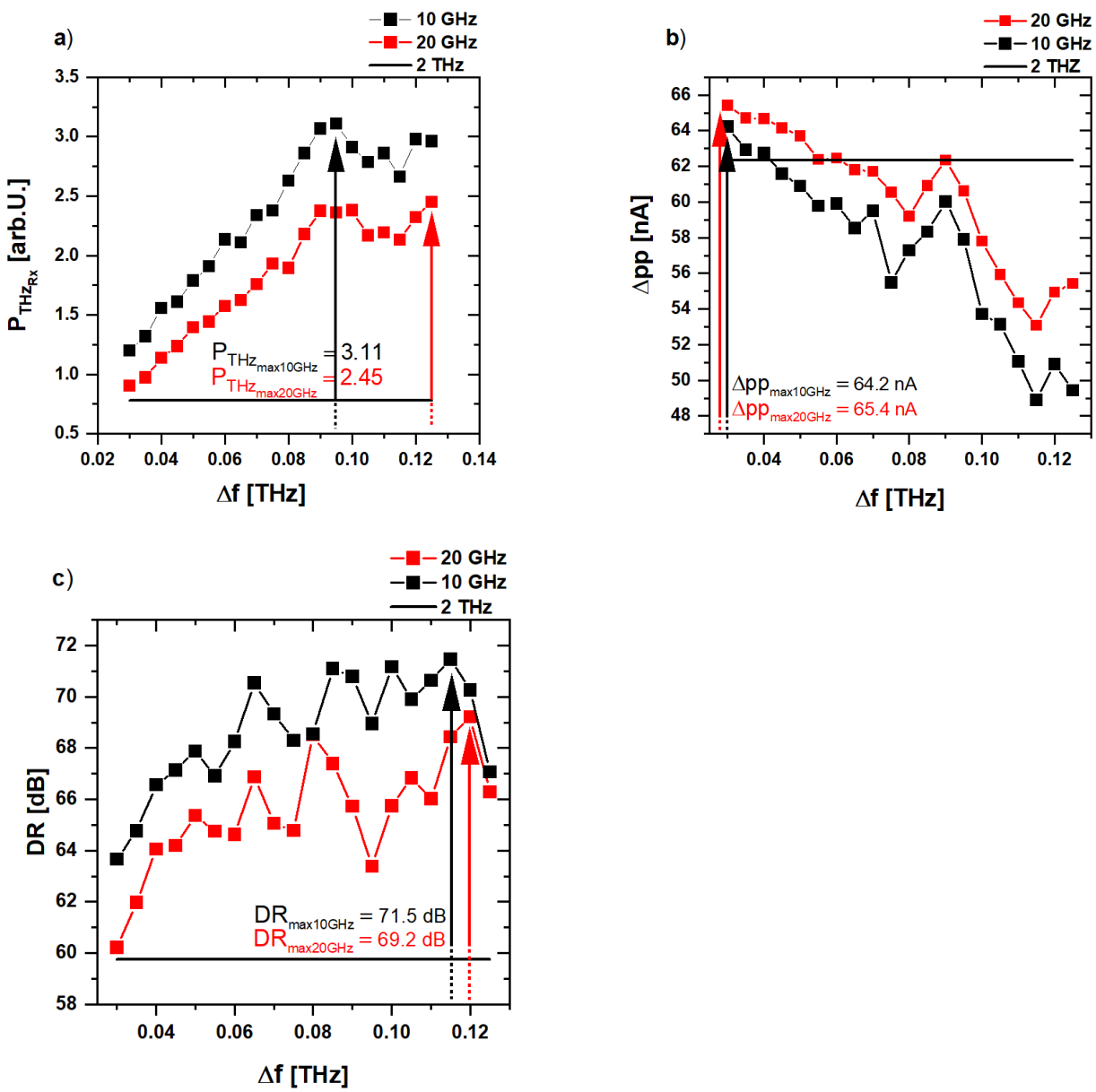

Figure 6. (a) Calculated power of the shaped $2 \mathrm{THz}$ bandpass (black line) with $10 \mathrm{GHz}$ (black squares) and $20 \mathrm{GHz}$ (red squares) feature width for different filter periods $\Delta \mathrm{f}$. (b) $\Delta \mathrm{pp}$ as a function of the filter period for a $10 \mathrm{GHz}$ and $20 \mathrm{GHz}$ feature width. (c) DR for the same feature width; a larger $\Delta \mathrm{f}$ results in a higher peak DR.

Figure 6a shows a sharp increase in the calculated power for the structured spectrum, which correlates with the filter period $\Delta f$. Regardless of feature size, the calculated power of the structured spectrum is always greater than the power from the continuous bandpass filtered spectrum. In addition, a smaller feature width results in higher power. For a feature width of $10 \mathrm{GHz}$ and a filter period of $0.095 \mathrm{THz}$, a power increase by a factor of 4 is observed. From the point of view of a mode-locked laser, this behavior is counterintuitive. A dense mode spectrum, i.e., a small free spectral range or filter period, results in pulses with a higher peak power. Since a high peak power is considered beneficial for THz-TDS systems, a different explanation is required for a THz-CCS system. If you think of the continuous spectrum as a spectrum of discrete lines with an infinitesimally small spacing, you get many mixing products with a very small difference frequency. These low-frequency components can neither be radiated nor detected by the antenna structure. Consequently, 
part of the optical power does not contribute to the terahertz generation. From this point of view, it becomes obvious why the structuring of the optical spectrum increases the radiated power: less power is used for non-radiable frequency components. The same is true for the filter period and feature width. Within a feature, there are also non-radiable difference frequency components. This effect is more pronounced for $20 \mathrm{GHz}$ than for $10 \mathrm{GHz}$ and explains the reduced calculated power. If the number of features is also reduced, which corresponds to an increase in the filter period, the low-frequency components become correspondingly fewer and the radiated or detected power is increased.

In Figure 6b, it can be seen that a comb structure can only outperform the continuous bandpass in terms of $\Delta p p$, if the number of peaks is high, respectively, the filter period is small within the $2 \mathrm{THz}$ span. Further, measurements with $20 \mathrm{GHz}$ feature width show a broader range for the filter period where $\Delta \mathrm{pp}$ is larger than the continuous BP compared to the measurements with $10 \mathrm{GHz}$ feature width. The largest increase in $\Delta \mathrm{pp}$ was $2 \mathrm{nA}$ for $10 \mathrm{GHz}$ and $4 \mathrm{nA}$ for $20 \mathrm{GHz}$. Overall, the influence of the filter period on $\Delta$ pp can be considered small and detrimental. It should be noted that a better performance is obtained for a feature width of $20 \mathrm{GHz}$ than for $10 \mathrm{GHz}$ in contrast to the calculated power and DR. This effect can be explained if the feature width is understood as the optical line width. The line width is reciprocal to the coherence length. In this case, a high coherence length means a large FWHM of the envelope [cf. Figure 3a)]. This distributes the energy over a larger sampling window and explains the slightly lower amplitude for the $10 \mathrm{GHz}$ feature width measurements and the larger calculated terahertz power. For the limit case of the continuous spectrum, a single pulse in which the entire power is contained is obtained in the time-domain. Since here the power is lower than in the structured case for the reasons described above, the amplitude is larger only for large filter periods.

In Figure $6 c$, the DR for different filter periods is presented. In contrast to $\Delta p p$ as shown in Figure 6b, the structured spectra outperform the continuous bandpass over the complete filter period range for the dynamic range. The smallest increase of $0.5 \mathrm{~dB}$ can be seen for a $20 \mathrm{GHz}$ feature width and a $30 \mathrm{GHz}$ filter period, while the largest increase in DR can be achieved for a filter period of $125 \mathrm{GHz}$ and a feature width of $10 \mathrm{GHz}$. The DR increases to $71.5 \mathrm{~dB}$ compared to the $59.8 \mathrm{~dB}$ from the continuous $2 \mathrm{THz} \mathrm{BP}$. This is an impressive enhancement in dynamic range by $11.7 \mathrm{~dB}$. The increase in dynamic range cannot be explained by the increased power alone. The calculated power was only increased by a factor of 4, while an increase in DR of $11.7 \mathrm{~dB}$ is associated with an increase in the signal-to-noise ratio by a factor of 14. It follows that structuring the spectrum not only increases the generated power, but also reduces the noise of the system. As mentioned earlier, the discrete spectrum produces many mixing products with low frequencies that do not contribute to the signal but, as these measurements show, to the noise. As a conclusion for maximizing the $\Delta \mathrm{pp}$, it is better to use a small filter period with larger features. This leads to slightly better results than for the unstructured case. If the dynamic range needs to be improved, small features with large feature periods are more suitable. Compared to the continuous case, a structured optical spectrum will always improve the DR.

Analyzing the slope $\mathrm{m}$ and FWHM of the comb-like structures at three different feature widths $(5 \mathrm{GHz}, 10 \mathrm{GHz}$, and $20 \mathrm{GHz})$ at $15 \mathrm{~mW}$ optical power helps to point out the relationship between the spectral shape and the bandwidth. The measurements were done at $15 \mathrm{~mW}$ power per antenna because the EDFA was not able to amplify all optical spectra with $5 \mathrm{GHz}$ feature width to $20 \mathrm{~mW}$ per antenna. Figure 7a shows the calculated slope $\mathrm{m}$ of the terahertz spectra, as indicated in Figures $2 \mathrm{c}$ and $3 \mathrm{c}$ for different filter periods. A constant behavior for all three features widths can be seen. However, if the mean value of all slopes is considered, the continuous bandpass filtered case shows better results, while the unfiltered spectrum shows the best values for $\mathrm{m}$. This behavior can be explained as follows. Since $m$ corresponds to the bandwidth of the terahertz signal independently of the dynamic range, a high optical bandwidth favors a small slope. However, this positive effect of the unfiltered spectrum is cancelled out by the increased noise, as described above. 

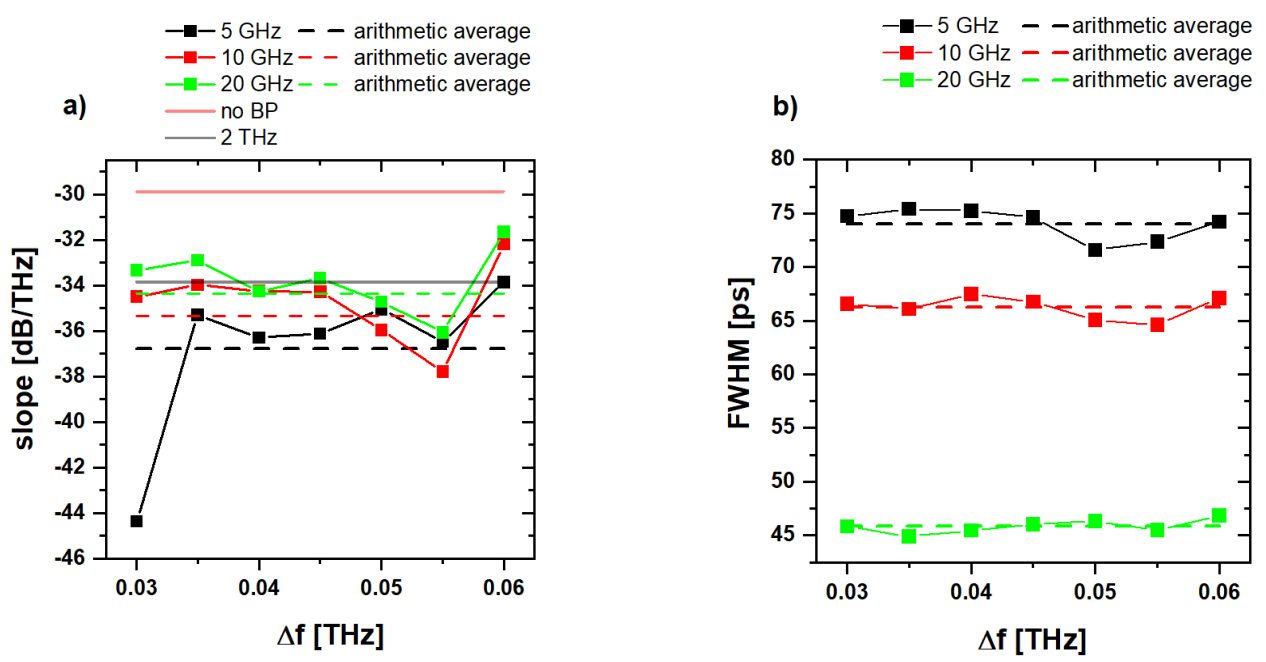

Figure 7. (a) Slope $\mathrm{m}$ at the receiver antenna with an incident optical power of $15 \mathrm{~mW}$. The arithmetic averages are rising for larger feature widths. The $5 \mathrm{THz}$ continuous bandpass filter which is given by the maximum bandpass of the POF has the lowest value for $\mathrm{m}$. For the $5 \mathrm{GHz}$ feature at $\Delta \mathrm{f}=0.03 \mathrm{THz}$, the optical power is too low to get a signal with a bandwidth higher than several hundreds of $\mathrm{GHz}$. (b) FWHM at the receiver antenna with an incident optical power of $15 \mathrm{~mW}$. The smaller the feature width of the optical spectrum, the larger the FWHM of the envelope of the time-domain trace.

So far, we have only assumed that the feature width correlates with the FWHM of the envelope in the time-domain. The coherence time as a function of linewidth is calculated as $t_{c}=1 / \Delta v$, where $t_{c}$ is correlated with the FWHM and $\Delta v$ is correlated with the feature width. Hence, the expected coherence time is 50 ps for $20 \mathrm{GHz}, 100$ ps for $10 \mathrm{GHz}$, and 200 ps for $5 \mathrm{GHz}$. Figure 7 shows the measured FWHM for the three feature widths. While the measured FWHM for $20 \mathrm{GHz}$ is close to the theoretical value at $45 \mathrm{ps,}$ there are large deviations for $10 \mathrm{GHz}$ and especially $5 \mathrm{GHz}$. There are two possible explanations for this discrepancy. First, the FWHM is only an estimate for the coherence time. Furthermore, the FWHM is not measured directly, as for example in a Fouriertransform infrared interferometer, but as a cross-correlation of the terahertz signal with the optical signal. Thus, effects at the detector are not taken into account. Second, due to the limited resolution, the feature width applied to the POF is not necessarily the feature width that is generated. The error becomes larger with smaller feature width. Nevertheless, this proves the correlation between the coherence length and the FWHM in the time-domain. A small feature width leads to a long coherence length and hence a larger FWHM. The extreme case is a mode-locked laser with an optical mode width on the order of a few MHz [18], which leads to a terahertz pulse train with constant amplitudes [19]. Further, it helps to understand why a smaller feature width increases the DR even if the $\Delta p p$ is reduced: within the broader envelope more pulses are included. Since all pulses contain the same information, an averaging effect occurs in the frequency-domain which increase the DR. All values are summarized in Table 2.

Table 2. Summary of the results in Figure 7. Mean slope and mean FWHM with respect to the POF parameters. The first two entries represent the $2 \mathrm{THz}$ continuous bandpass and the case if no filter is applied $(5 \mathrm{THz})$.

\begin{tabular}{cccc}
\hline Power & Feature Width & Mean FWHM & Mean Slope \\
\hline $15 \mathrm{~mW}$ & $2 \mathrm{THz}$ & $0.9 \mathrm{ps}$ & $-29.9 \mathrm{~dB} / \mathrm{THz}$ \\
$15 \mathrm{~mW}$ & $5 \mathrm{THz}$ & $0.9 \mathrm{ps}$ & $-33.8 \mathrm{~dB} / \mathrm{THz}$ \\
$15 \mathrm{~mW}$ & $5 \mathrm{GHz}$ & $74 \mathrm{ps}$ & $-36.8 \mathrm{~dB} / \mathrm{THz}$ \\
$15 \mathrm{~mW}$ & $10 \mathrm{GHz}$ & $66.3 \mathrm{ps}$ & $-35.4 \mathrm{~dB} / \mathrm{THz}$ \\
$15 \mathrm{~mW}$ & $20 \mathrm{GHz}$ & $45.8 \mathrm{ps}$ & $-34.4 \mathrm{~dB} / \mathrm{THz}$ \\
\hline
\end{tabular}




\subsection{Simulation Results}

To support the interpretation of the experimental results from the previous section, the system has been simulated in MATLAB with a simple numerical model. The electric field $E_{S L D}$ emitted by the SLD is modeled as band-limited white noise. The structuring of the optical spectrum is considered by numerically filtering the signal with a periodic ideal bandpass filter whose passband-width and period are matched to the experimentally implemented shapes. It was shown by Kolpatzeck et al. that the detected complex terahertz spectrum $S_{T H z}(\omega)$ in a MLLD-driven THz-CCS system is proportional to the magnitude $\left|S_{I I}(\omega)\right|$ of the Fourier transform of the intensity autocorrelation function $\mathrm{R}_{\mathrm{II}}(\tau)$ of the optical signal multiplied with the system's complex transfer function $H_{S y s}(\omega)$ [15]. It is reasonable to assume that this model is also valid in a SLD-driven THz-CCS-system. The intensity autocorrelation function $\mathrm{R}_{\mathrm{II}}(\tau)$ is numerically determined from the optical intensity $I_{S L D}(t)=\left|E_{S L D}(t)\right|^{2}$. The resulting terahertz spectrum can then be derived from:

$$
S_{T H z}(\omega)=H_{S y s}(\omega) \cdot\left|S_{I I}(\omega)\right|
$$

$S_{T H z}(\omega)$ is the complex terahertz spectrum. $H_{S y s}(\omega)$ is the complex transfer function (system dependent). $\left|S_{I I}(\omega)\right|$ stands for the magnitude of the Fourier transform of the intensity autocorrelation function $\mathrm{R}_{\mathrm{II}}(\tau)$. The transfer function was measured with a commercially available continuous-wave terahertz frequency-domain spectroscopy system (Toptica TeraScan 1550). The simulated photocurrent in the time-domain is calculated by inverse Fourier transform of $S_{T H z}(\omega)$. To compare the measurement with the simulation, the same time range relative to the maximum of the simulated photocurrent as in the measurement is considered. Moreover, the same signal processing and analysis steps are applied to the simulated data as to the measurement data. The comparison of the measurement and simulation can be seen in Figure 8 for the parameters $P_{T H z}, \Delta \mathrm{pp}$, and DR, respectively. To effectively compare the effects of the structuring of the optical spectrum, the data for $P_{T H z}, \Delta \mathrm{pp}$, and DR are normalized to the measurements for the $2 \mathrm{THz}$ reference bandpass structure. The solid black line in Figure 8 represents the value for the $2 \mathrm{THz}$ reference measurements. The simulation shows a good agreement with the measurement results. For the feature periods in the range from $0.03-0.9 \mathrm{THz}$, the simulation results match the experiment nearly perfectly for $P_{T H z}$ with a feature width of $20 \mathrm{GHz}$, while it shows a $\sim 33 \%$ difference in the $10 \mathrm{GHz}$ feature width case. The graph for $\Delta \mathrm{pp}$ shows again a strong deviation for filter periods bigger than $0.8 \mathrm{THz}$. However, the rest of the data match the measurement results quite well. In Figure $8 \mathrm{c}$, the DR is plotted and the deviation between measurement and simulation is similar for both feature widths and lies around $5 \%$ lower than the measurement data. These results correspond to the fact that there are effects in a THz-CCS system which are not included in our model. However, we can predict the DR of a THz-CCS system with this numerical approach with a $5 \%$ accuracy. 

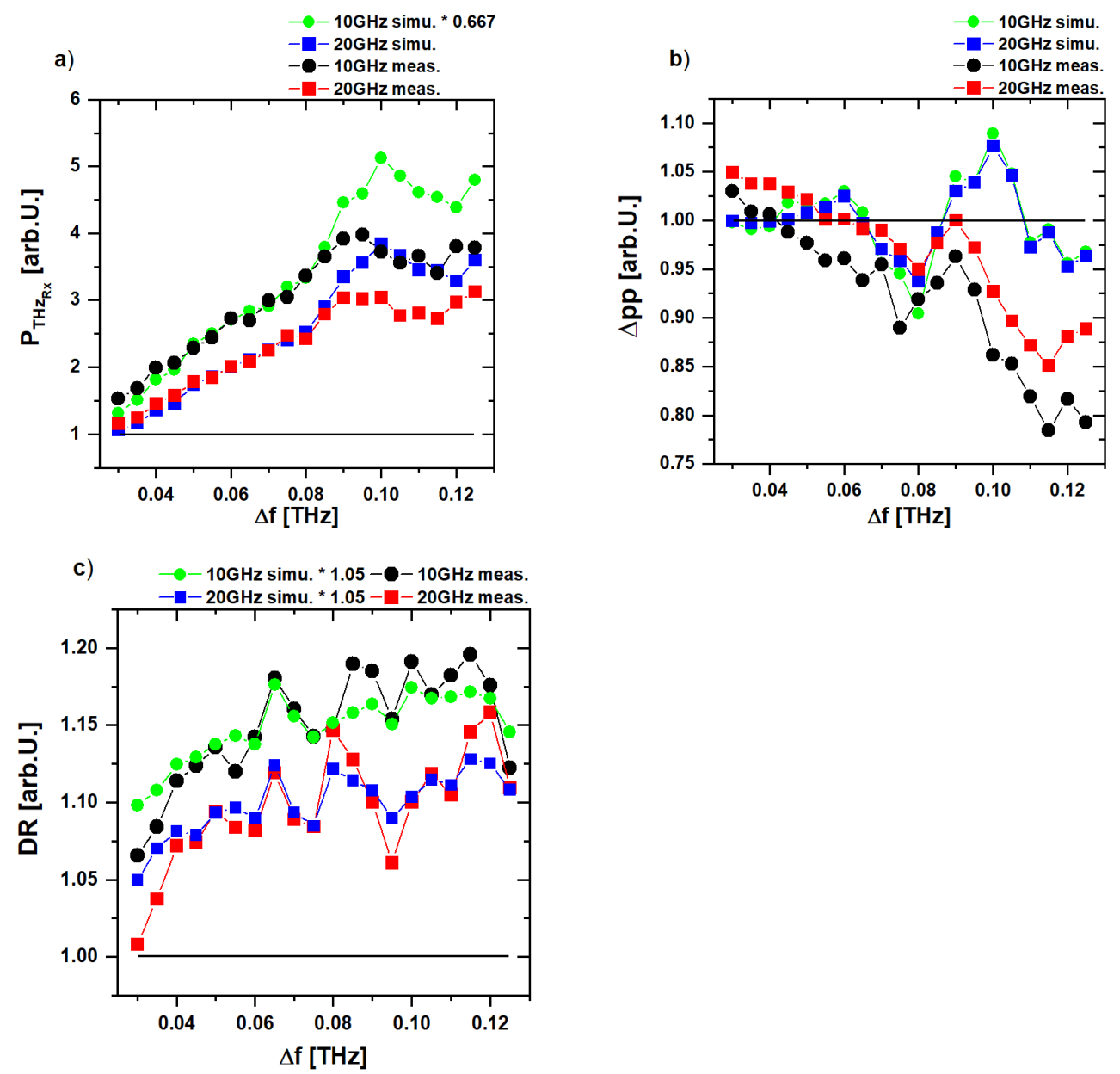

Figure 8. (a) Calculated power of the shaped $2 \mathrm{THz}$ bandpass (black line) with $10 \mathrm{GHz}$ (black squares) and $20 \mathrm{GHz}$ (red squares) feature width for different filter periods $\Delta \mathrm{f}$. (b) $\Delta \mathrm{pp}$ as a function of the filter period for a $10 \mathrm{GHz}$ and $20 \mathrm{GHz}$ feature width. (c) DR for the same feature width; a larger $\Delta \mathrm{f}$ results in a higher peak DR.

\section{Conclusions}

A THz-CCS system was characterized with special interest in the parameters dynamic range, peak-to-peak amplitude, THz-power, and bandwidth. The objective was to get a better understanding of the influence of the shape of the optical spectrum on these parameters. We demonstrated that structuring of the optical spectrum increases the dynamic range and bandwidth of the signal. Small feature widths with large filter periods are favorable if an increase in the dynamic range of the system is required. However, the increase comes at the cost of a discrete spectrum which limits the spectral resolution to the filter period. If a high peak-to-peak amplitude is desired, it is favorable to use continuous shapes because they show the highest peak-to-peak amplitude in connection with a high bandwidth and a continuous spectrum. However, the gain in peak-to-peak amplitude is negligible compared to the loss in dynamic range. The structuring of the spectrum comes at a price: due to the periodicity of the spectrum, the frequency resolution is reduced to the filter period. Comparing the results to modern continuous wave terahertz systems, a lack of bandwidth becomes apparent. The emitter and the receiver have a bandwidth up to $2.75 \mathrm{THz}$ for continuous wave excitation [13] and, in a recent publication, Liebermeister et al. (2021) demonstrated a bandwidth of up to $4 \mathrm{THz}$ after averaging the signal 250,000 times, with the same antenna configuration [20]. However, distributed feedback lasers, which have a narrow linewidth, were used as light sources. In this work, a single incoherent broadband light source was used, reducing the bandwidth to about $1.8 \mathrm{THz}$. The lower bandwidth can be explained by the wider linewidth of the filtered spectrum and lower averaging. It 
follows that for future laser diode-based terahertz systems (mode-locked or not), special attention must be paid to the optical linewidth. Further, it explains the limited bandwidth of SLD-driven THz-CCS systems.

In summary, a concept for a THz-CCS system is presented which can be used for prototyping bandpass filters and laser concepts. The results represent a technique to further improve the system performance regarding terahertz power, peak-to-peak amplitude, and peak dynamic range. All measurements were validated with a numerical model. The results give more room for system development which will ultimately lead to compact, mobile, and robust THz-CCS systems which can be optimized for a specific application.

Author Contributions: K.-H.T.: system design, experiment design, data acquisition, preparation, data analysis, editing and writing; K.K.: theoretical modelling, simulation results, data analysis; V.C.: measurement software; B.F.: Third Supervisor, funding acquisition; S.E.: Second Supervisor, review editing; J.C.B.: First Supervisor, experiment design, review editing. All authors have read and agreed to the published version of the manuscript.

Funding: Funded by the Deutsche Forschungsgemeinschaft (DFG, German Research Foundation) -Project-ID 287022738 TRR 196.

Data Availability Statement: The data presented in this study are available on request from the corresponding author.

Acknowledgments: We acknowledge support by the Open Access Publication Fund of the University of Duisburg-Essen.

Conflicts of Interest: The authors declare no conflict of interest.

\section{Appendix A}

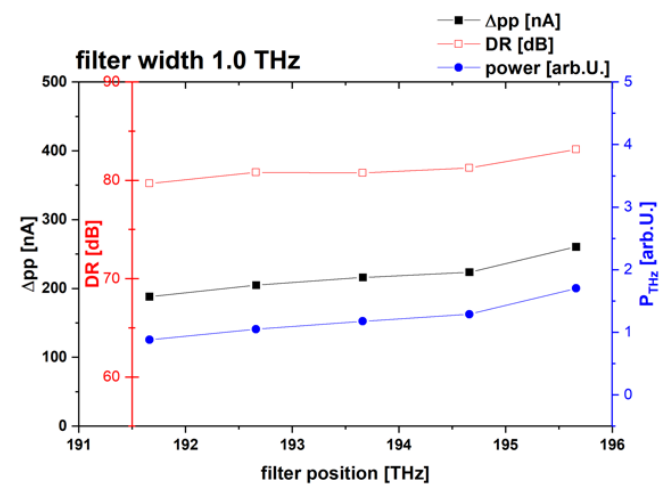

Figure A1. Peak-to-peak amplitude, dynamic range, and calculated terahertz power for a $1 \mathrm{THz}$ broad filter moved to six different positions of the SLD spectrum.

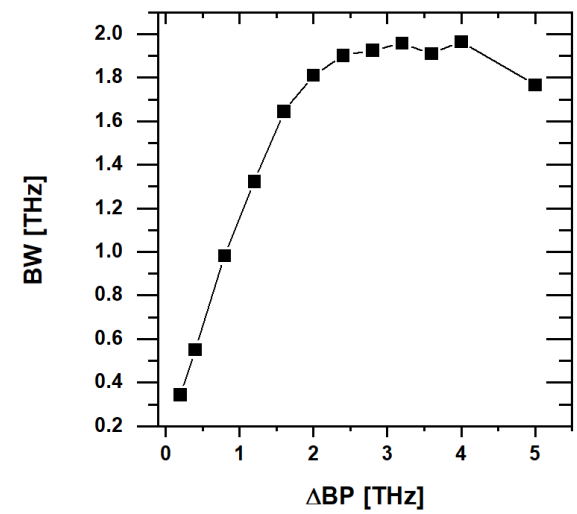

Figure A2. Detected bandwidth of the terahertz signal as a function of the width of the optical bandpass filter. A saturation occurs around $2 \mathrm{THz}$. 


\section{References}

1. Morikawa, O.; Tonouchi, M.; Hangyo, M. A cross-correlation spectroscopy in subterahertz region using an incoherent light source. Appl. Phys. Lett. 2000, 76, 1519. [CrossRef]

2. Morikawa, O.; Fujita, M.; Hangyo, M. Improvement of signal-to-noise ratio of a subterahertz spectrometer using a continuouswave multimode laser diode by single-mode fiber optics. Appl. Phys. Lett. 2004, 85, 881-883. [CrossRef]

3. Shibuya, K.; Tani, M.; Hangyo, M.; Morikawa, O.; Kan, H. Compact and inexpensive continuous-wave subterahertz imaging system with a fiber-coupled multimode laser diode. Appl. Phys. Lett. 2007, 90, 161127. [CrossRef]

4. Scheller, M.; Koch, M. Terahertz quasi time domain spectroscopy. Opt. Express 2009, 17, 17723. [CrossRef] [PubMed]

5. Morikawa, O.; Fujita, M.; Takano, K.; Hangyo, M. Sub-terahertz spectroscopic system using a continuous-wave broad-area laser diode and a spatial filter. J. Appl. Phys. 2011, 110, 063107. [CrossRef]

6. Molter, D.; Wagner, A.; Weber, S.; Jonuscheit, J.; Beigang, R. Combless broadband terahertz generation with conventional laser diodes. Opt. Express 2011, 19, 5290. [CrossRef] [PubMed]

7. Kohlhaas, R.B.; Rehn, A.; Nellen, S.; Koch, M.; Schell, M.; Dietz, R.J.B.; Balzer, J.C. Terahertz quasi time-domain spectroscopy based on telecom technology for $1550 \mathrm{~nm}$. Opt. Express 2017, 25, 12851. [CrossRef] [PubMed]

8. Merghem, K.; Busch, S.F.; Lelarge, F.; Koch, M.; Ramdane, A.; Balzer, J.C. Terahertz Time-Domain Spectroscopy System Driven by a Monolithic Semiconductor Laser. J. Infrared Millim. Terahertz Waves 2017, 38, 958-962. [CrossRef]

9. Rehn, A.; Mikerov, M.; Preu, S.; Koch, M.; Balzer, J.C. Enhancing the performance of THz quasi time-domain spectroscopy systems by low duty cycle laser operation. Opt. Express 2018, 26, 32758. [CrossRef] [PubMed]

10. Rehn, A.; Kohlhaas, R.; Globisch, B.; Balzer, J.C. Increasing the THz-QTDS Bandwidth from 1.7 to 2.5 THz through Optical Feedback. J. Infrared Millim. Terahertz Waves 2019, 40, 1103-1113. [CrossRef]

11. Tonder, S.C.; Kolpatzeck, K.; Liu, X.; Rumpza, S.; Czylwik, A.; Balzer, J.C. A compact THz quasi TDS system for mobile scenarios In Proceedings of the 2019 Second International Workshop on Mobile Terahertz Systems (IWMTS), Bad Neuenahr, Germany, 1-3 July 2019; pp. 1-5.

12. Molter, D.; Kolano, M.; von Freymann, G. Terahertz cross-correlation spectroscopy driven by incoherent light from a superluminescent diode. Opt. Express 2019, 27, 12659. [CrossRef] [PubMed]

13. Deninger, A.J.; Roggenbuck, A.; Schindler, S.; Preu, S. $2.75 \mathrm{THz}$ tuning with a triple-DFB laser system at $1550 \mathrm{~nm}$ and InGaAs photomixers. J. Infrared Millim. Terahertz Waves 2014, 36, 269-277. [CrossRef]

14. Preu, S. A Unified Derivation of the Terahertz Spectra Generated by Photoconductors and Diodes. J. Infrared Millim. Terahertz Waves 2014, 35, 998-1010. [CrossRef]

15. Kolpatzeck, K.; Liu, X.; Tybussek, K.-H.; Häring, L.; Zander, M.; Rehbein, W.; Moehrle, M.; Czylwik, A.; Balzer, J.C. Systemtheoretical modeling of terahertz time-domain spectroscopy with ultra-high repetition rate mode-locked lasers. Opt. Express 2020 28, 16935. [CrossRef] [PubMed]

16. Globisch, B.; Dietz, R.J.B.; Göbel, T.; Schell, M.; Bohmeyer, W.; Müller, R.; Steiger, A. Absolute terahertz power measurement of a time-domain spectroscopy system. Opt. Lett. 2015, 40, 3544. [CrossRef] [PubMed]

17. Nellen, S.; Ishibashi, T.; Deninger, A.; Kohlhaas, R.B.; Liebermeister, L. Experimental Comparison of UTC-and PIN-Photodiodes for Continuous-Wave Terahertz Generation. J. Infrared Millim. Terahertz Waves 2020, 41, 343-354. [CrossRef]

18. Zander, M.; Rehbein, W.; Moehrle, M.; Breuer, S.; Franke, D.; Schell, M.; Kolpatzeck, K.; Balzer, J.C. High performance BH InAs/InP QD and InGaAsP/InP QW mode-locked lasers as comb and pulse sources. In Proceedings of the Optical Fiber Communication Conference (OFC) 2020, San Diego, CA, USA, 8-12 March 2020; OSA: Washington, DC, USA, 2020; pp. 782-784.

19. Kolpatzeck, K.; Liu, X.; Häring, L.; Balzer, J.C.; Czylwik, A. Ultra-high repetition rate terahertz time-domain spectroscopy for micrometer layer thickness measurement. Sensors 2021, 21, 5389. [CrossRef] [PubMed]

20. Liebermeister, L.; Nellen, S.; Kohlhaas, R.B.; Lauck, S.; Deumer, M.; Breuer, S.; Schell, M.; Globisch, B. Optoelectronic frequencymodulated continuous-wave terahertz spectroscopy with $4 \mathrm{THz}$ bandwidth. Nat. Commun. 2021, 12, 1071. [CrossRef] [PubMed] 